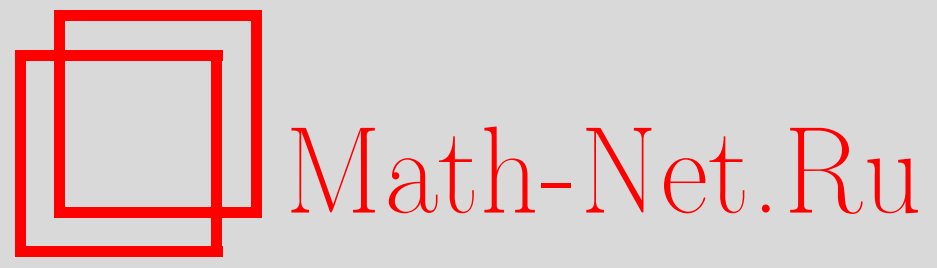

М. М. Глухов (мл.), О кодах Гоппы на одном семействе полей алгебраических чисел, Дискрет. матем., 2001, том 13, выпуск 2, 14-34

DOI: https://doi.org/10.4213/dm281

Использование Общероссийского математического портала Math-Net.Ru подразумевает, что вы прочитали и согласны с пользовательским соглашением http://www . mathnet.ru/rus/agreement

Параметры загрузки:

IP: 54.237 .206 .68

26 апреля 2023 г., 12:53:13

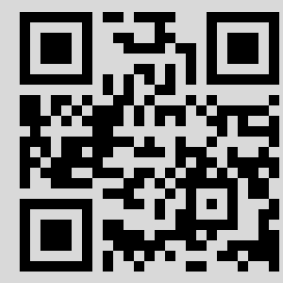


УДК 519.4

\section{О кодах Гоппы на одном семействе полей алгебраических чисел}

(c) 2001 г. М. М. Глухов (мл.)

В работе описаны свойства геометрических кодов Гопты на кривой, заданной уравнением

$$
y^{s}=\left(x^{q^{(n-u) / 2}-1}+1\right)^{a}\left(x^{q^{(n+u) / 2}-1}+1\right)^{b}
$$

над конечным полем $K=F_{q^{n}}$ при произвольных нечетном $q, n>1$, где $s=a+b$, $s \mid q-1, u=1$ при нечетном $n$ и $u=2$ при четном $n$. Находится число и вид

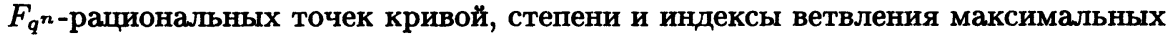
идеалов колец дискретного нормирования поля $K(x, y)$ и соответствующие им показатели. В некоторых случаях находятся базисы кодов.

\section{1. Введение}

Максимальная длина геометрического кода Гоппы на кривой $V$, заданной уравнением

$$
y^{s}=f(x)
$$

над конечным полем $K$, равна числу $K$-рациональных точек на этой кривой (см., например, [7]). В связи с этим представляют особый интерес такие многочлены $f(x) \in K[x]$, при которых уравнение (1) имеет сравнительно большое число решений. $\mathrm{K}$ таким многочленам относятся, в частности, рассмотренные в [1] многочлены вида

$$
\left(\mu x+(\mu x)^{q^{(n-u) / 2)}}\right)^{a}\left(\mu x+(\mu x)^{q^{(n+u) / 2)}}\right)^{b}
$$

над полем $K=F_{q^{n}}$, где $q, a, b, n \in \mathbf{N}, q$ нечетно, $n>1, a+b=s, \mu \in K^{*}, u=1$ при нечетном $n, u=2$ при четном $n$. В [4] был доказан ряд утверждений о существовании кодов Гоппы с достаточно хорошими параметрами на кривых (1) с многочленом (2). Более глубокое изучение кодов на такой кривой требует перехода от кривой (1) к полю $K(x, y)$ алгебраических функций, заданному уравнением (1). Связи между алгебраическими кривыми и полями алгебраических функций хорошо известны (см., например, [7]). При переходе от кривой (1) к полю $K(x, y) K$-рациональным точкам кривой соответствуют максимальные идеалы первой степени колец дискретного нормирования поля $K(x, y)$. В то же время построение кодов на кривой (1) 
и изучение их свойств требуют знания максимальных идеалов больших степеней и умения вычислять значения и показатели элементов поля $K(x, y)$ относительно этих идеалов. Для решения последней задачи необходимо описание решений уравнения (1) и неприводимых над $K$ делителей многочлена $f(x)$.

Для многочленов вида (2) последние задачи мало зависят от параметра $\mu$ и от множителя $(\mu x)^{s}$. А число точек на кривой при удалении этого множителя даже увеличивается. В связи с этим всюду далее рассматриваются лишь многочлены вида

$$
f(x)=\left(x^{q^{(n-u) / 2}-1}+1\right)^{a}\left(x^{q^{(n+u) / 2}-1}+1\right)^{b} .
$$

В параграфе 2 данной статьи описываются при нечетном $q$ все делители таких многочленов, неприводимые над над полями $F_{q}$ и $F_{q^{n}}$, находятся формулы для числа неприводимых делителей заданной степени. В случае четного $q$ обе эти задачи решаются тривиально. Здесь же находится число и вид $F_{q^{n}}$-рациональных точек на кривой (1) с многочленами вида (3). В параграфе 3 на основе результатов параграфа 2 и известных фактов о полях алгебраических функций [7] находятся степени и индексы ветвления максимальных идеалов колец дискретного нормирования поля $K(x, y)$ и соответствующие им показатели. В параграфе 4 результаты параграфов 2 и 3 применяются к построению кодов на рассматриваемых полях. В частности, уточняются параметры кодов, указанные в [4], в некоторых случаях находятся базисы кодов.

\section{2. О неприводимых делителях многочленов}

\section{и решениях уравнения}

В данном пункте будут использоваться следующие обозначения: $q$ - степень простого нечетного числа; $F_{q}$ - поле из $q$ элементов, $F_{q^{n}}$ - его расширение степени $n$, $\exp _{2}(x)$ - максимальная степень числа 2 , на которую делится целое число $x \neq 0$; $m \in \mathbf{N}, m=2^{k} m_{1}$, где $m_{1}$ - нечетно; $\operatorname{ord}(\alpha)$ - порядок корня $\alpha$ многочлена в мультипликативной группе поля разложения этого многочлена, $e=\exp _{2}\left(2\left(q^{m / 2}-1\right)\right), \phi$ - функция Эйлера, $\Phi(m)$ - число неприводимых унитарных многочленов степени $m$ из кольца $F_{q}[x]$ (всех, при $m>1$ и всех, кроме $x$, при $m=1$ ). В ходе доказательств без ссылок будут использоваться следующие известные факты из теории конечных полей [3]. Многочлен $x^{q^{n}}-x$ есть произведение всех неприводимых над $F_{q}$ унитарных многочленов, степени которых делят $n$; многочлен $f(x)$ степени $m$ неприводим над $F_{q}$ в том и только том случае, когда он делит многочлен $x^{q^{m}}-x$ и не делит многочлен $x^{q^{\prime}}-x$ при $r<m$; все корни $\alpha$ неприводимого многочлена степени $m$ над $F_{q}$ имеют один и тот же порядок и характеризуются тем, что ord $(\alpha)$ делит число $q^{m}-1$ и не делит число вида $q^{r}-1$ при $r<m$. Кроме того, часто будет использоваться следующее очевидное утверждение.

Лемма 1. Для любого натуралъного числа $r$ имеет место равенство

$$
\exp _{2}\left(q^{r}-1\right)=\exp _{2}(q-1)+\exp _{2}(r)+c
$$

әде $c=0$, если $\exp _{2}(q-1)>1$ или $\exp _{2}(r)=0, u c=\exp _{2}(q+1)-1$ в осталънъх cлугаяx. 
Для описания неприводимьх делителей всех многочленов вида (2) над полем $F_{q}$ достаточно сделать это для многочлена

$$
g_{u}(x)=x^{q^{(n+u) / 2}}+x
$$

где $u=1$ или -1 при нечетном $n$ и $u=2$ или -2 при четном $n$.

Теорема 1. Неприводимый над полем $F_{q}$ многочлея $h(x)$ степени $m$ делит $g_{u}(x)$ в том и только том случае, когда выполняяотся условия

$$
\begin{gathered}
m \mid n+u, \quad m \nmid(n+u) / 2, \\
\operatorname{ord}(\alpha) \mid 2\left(q^{m / 2}-1\right),
\end{gathered}
$$

где $\alpha-$ корень $h(x)$.

При въполнении условий (4) $u(5)$ число $X_{m}$ неприводимъх над полем $F_{q}$ унитарньх делителей степени $m$ многочлена $g_{u}(x)$ равно

$$
X_{m}=\sum_{i=1}^{k} \frac{1}{2^{i}} \Phi\left(\frac{m}{2^{i}}\right) .
$$

Доказательство. Докажем первое утверждение теоремы. Из неприводимости многочлена $h(x)$ над $F_{q}$ следует, что

$$
\begin{aligned}
\alpha^{q^{m}-1} & =1 \\
\alpha^{q^{r}-1} & =1 \Longleftrightarrow m \mid r, \quad r \in \mathbf{N} .
\end{aligned}
$$

Пусть $h(x) \mid g_{u}(x)$. Тогда $\alpha$ - корень $g_{u}(x)$, то есть

$$
\begin{aligned}
& \alpha^{q^{(n+u) / 2}-1}=-1, \\
& \alpha^{2\left(q^{(n+u) / 2}-1\right)}=1 .
\end{aligned}
$$

Из (10), учитывая, что $q$ нечетно, получаем, что $\alpha^{\left(q^{(n+u) / 2}-1\right)\left(q^{(n+u) / 2}+1\right)}=1$, то есть

$$
\alpha^{q^{n+u}-1}=1
$$

Из (11), в частности, следует, что $F_{q^{n+u}}$ есть поле разложения многочлена $h(x)$. Из (11) и (8) получаем, что $m \mid n+u$. Если $m \mid(n+u) / 2$, то $q^{m}-1 \mid q^{(n+u) / 2}-1$, и в силу (7), $\alpha^{q^{(n+u) / 2}-1}=1$, вопреки условию (9). Таким образом, условие (4) выполнено.

Докажем (5). Из (7) и (10) следует, что $\alpha^{d}=1$, где $d=\left(q^{m}-1,2\left(q^{(n+u) / 2}-1\right)\right)$. Найдем $d$. Из условия (4) следует, что

$$
\exp _{2}((n+u) / 2)=\exp _{2}(m / 2)<\exp _{2}(m)
$$

Отсюда, в силу леммы 1 , получаем, что

$$
\exp _{2}\left(q^{(n+u) / 2}-1\right)=\exp _{2}\left(q^{m / 2}-1\right)<\exp _{2}\left(q^{m}-1\right)
$$

Следовательно,

$$
d=2\left(q^{m}-1, q^{(n+u) / 2}-1\right)=2\left(q^{(m,(n+u) / 2)}-1\right)=2\left(q^{m / 2}-1\right),
$$


и из равенства $\alpha^{d}=1$ получаем либо $\alpha^{q^{m / 2}-1}=-1$, либо $\alpha^{q^{m / 2}-1}=1$, причем последнее противоречит условию (8). Таким образом, $\alpha^{2\left(q^{m / 2}-1\right)}=1$ и $\operatorname{ord}(\alpha) \mid 2\left(q^{n / 2}-1\right)$.

Обратно, пусть выполнены условия (4) и (5). Из условия $m \mid n+u$ и равенства в (12) следует, что

$$
q^{(n+u) / 2}-1=\left(q^{m / 2}-1\right) c
$$

где $c$ нечетно. Тогда

$$
\alpha^{q^{(n+u) / 2}-1}=\alpha^{\left(q^{m / 2}-1\right) c}=(-1)^{c}=-1,
$$

то есть $\alpha$ - корень $g_{u}(x)$. Следовательно, $h(x) \mid g_{u}(x)$, и первое утверждение теоремы доказано.

Докажем теперь (6). Зафиксируем число $m \in \mathbf{N}$, удовлетворяющее условию (4), и найдем число $X_{m}$. Из первого утверждения теоремы видно, что $X_{m}=N / m$, где $N$ - число элементов $\beta$ поля $F_{q^{n+u}}$, удовлетворяющих (5) и условию $\operatorname{ord}(\beta) \nmid q^{r}-1$ при $0<r<m$. Для отыскания числа $N$ рассмотрим сначала множество $M$ всех делителей числа $2\left(q^{m / 2}-1\right)$, не являющихся делителями числа $q^{r}-1$ при $0<r<m$. Если $\exp _{2}\left(2\left(q^{m / 2}-1\right)\right)=e$ и $P=\left\{c_{1}, \ldots, c_{t}\right\}-$ множество всех нечетньх делителей $q^{m / 2}-1$, то $M$ может содержать лишь некоторые числа вида $2^{e} c_{j}$. Учитывая (5), нетрудно заметить, что условие, состоящее в том, что $2^{e} c_{j} \mid q^{r}-1$ при некотором $0<r<m$, эквивалентно условию, состоящему в том, что $2^{e-1} c_{j} \mid q^{r_{1}}-1$ при некотором $0<r_{1}<m / 2$ (при фиксированном $r\left(r_{1}\right)$ в качестве $r_{1}(r)$ можно взять $(r, m / 2)$ $\left.\left(2 r_{1}\right)\right)$. Следовательно,

$$
M=\left\{2^{e} c_{j}: j \in\{1, \ldots, t\}, 2^{e-1} c_{j} \nmid q^{r_{1}}-1, r_{1}<m / 2\right\} .
$$

Разобьем $P$ на три подмножества

$$
P_{1}=\left\{c_{11}, \ldots, c_{1 k_{1}}\right\}, \quad P_{2}=\left\{c_{21}, \ldots, c_{2 k_{2}}\right\}, \quad P_{3}=\left\{c_{31}, \ldots, c_{3 k_{3}}\right\}
$$

так, что $c_{1 i} \nmid q^{r}-1$ при $0<r<m / 2 ; c_{2 j} \mid q^{r}-1$ при $0<r<m / 2$ и минимальное из таких $r$ имеет вид $r_{j}=m / 2^{\nu_{j}} ; c_{3 j} \mid q^{r}-1$ при $0<r<m / 2$ и минимальное из таких $r$ не имеет вида $m / 2^{\nu}$. Для упрощения записей обозначим $R$ условие, состоящее в том, что $c \nmid q^{r}-1$ при $0<r<m / 2$, а его отрицание будем обозначать $\bar{R}$.

Докажем следующее утверждение.

Лемма 2. Если $с \in P_{1}$, то числа $2^{j}$ с удовлетворяют условия $R$ при $j=0, \ldots, e-1$.

Если $c \in P_{2}$, то иисла $2^{j}$ с удовлетворяют условию $\bar{R}$ при $j=0,1, \ldots, h$ и условию $R$ при $j=h+1, \ldots, e-1$, где $h=\exp _{2}\left(q^{m / 4}-1\right)$.

Если $c \in P_{3}$, то числа $2^{j}$ с удовлетворяют условию $\bar{R}$ при $j=0,1, \ldots, e-1$.

Доказательство. Первое утверждение очевидно.

Докажем второе утверждение. Так как минимальное $r<m / 2$, при котором $c$ делит $q^{r}-1$, имеет вид $m / 2^{j}$, число $2^{j} c$ удовлетворяет условию $\bar{R}$ тогда и только тогда, когда $2^{j} c \mid q^{m / 4}-1$. Значит, максимальное из таких чисел $j$ совпадает с $\exp _{2}\left(q^{m / 4}-1\right)$, и утверждение доказано.

Докажем третье утверждение. Пусть минимальное $r<m / 2$, при котором $c$ делит $q^{r}-1$, имеет вид $r=2^{\gamma} r_{1},\left(r_{1}, 2\right)=1$, и $m=2^{k} m_{1},\left(m_{1}, 2\right)=1$. Поскольку $c \mid q^{m / 2}-1$, то $r \mid m / 2$. Следовательно, $r_{1} \mid m_{1}, \gamma \leqslant k-1$. Учитывая, что $c \in P_{3}$, получаем, что $r_{1}<m_{1}$ и, значит, $2^{k-1} r_{1}<m / 2$. Так как $c \mid q^{2^{k-1} r_{1}}-1$ и

$$
\exp _{2}\left(q^{2^{k-1} r_{1}}-1\right)=\exp _{2}\left(q^{m / 2}-1\right)=e-1
$$


то $2^{(e-1)} c \mid q^{2^{k-1} r_{1}}-1$, то есть $2^{j} c$ удовлетворяет условию $\bar{R}$ при $j=0, \ldots, e-1$. Лемма доказана.

Из леммы 2 следует, что порядками корней неприводимых делителей степени $m$ многочлена $g_{u}(x)$ являются лишь числа $2^{e} c_{1 i}, i=1, \ldots, k_{1}$, и $2^{e} c_{2 j}, j=1, \ldots, k_{2}$. Отсюда, учитывая, что в группе $F_{q^{n+u}}^{*}$ для каждого делителя $d \mid q^{n+u}-1$ существует ровно $\phi(d)$ элементов порядка $d$, получим, что

$$
X_{m}=\frac{1}{m}\left(\sum_{i=1}^{k_{1}} \phi\left(2^{e} c_{1 i}\right)+\sum_{i=1}^{k_{2}} \phi\left(2^{e} c_{2 i}\right)\right)
$$

Так как $\phi\left(2^{r} c\right)=2^{r-1} \phi(c)$ при $r>0$, то

$$
X_{m}=\frac{2^{e-1}}{m}\left(\sum_{i=1}^{k_{1}} \phi\left(c_{1 i}\right)+\sum_{i=1}^{k_{2}} \phi\left(c_{2 i}\right)\right)
$$

или, короче,

$$
X_{m}=\frac{2^{e-1}}{m}\left(S_{1}+S_{2}\right)
$$

где

$$
S_{1}=\sum_{i=1}^{k_{1}} \phi\left(c_{1 i}\right), \quad S_{2}=\sum_{i=1}^{k_{2}} \phi\left(c_{2 i}\right) .
$$

Вычислим $S_{1}+S_{2}$. Из леммы 2 следует, что множество всех делителей $D$ числа $q^{m / 2}-1$ с условием $R$ исчерпывается числами

$$
\begin{aligned}
& 2^{j} c_{1 i}, \quad i=1, \ldots, k_{1}, \quad j=0, \ldots, e-1, \\
& 2^{j} c_{2 i}, \quad i=1, \ldots, k_{2}, \quad j=h+1, \ldots, e-1 \text {. }
\end{aligned}
$$

Отсюда следует, что

$$
\frac{m}{2} \Phi\left(\frac{m}{2}\right)=\sum_{d \in D} \phi(d) .
$$

Используя свойства функции Эйлера, находим, что

$$
\frac{m}{2} \Phi\left(\frac{m}{2}\right)=2^{e-1} S_{1}+\left(2^{h}+\ldots+2^{e-2}\right) S_{2} .
$$

Далее мы вычислим $S_{2}$, а затем, с учетом формулы (13) найдем сумму $S_{1}+S_{2}$.

Для вычисления $S_{2}$ разобьем $P_{2}$ на подмножества $P_{2,0}, P_{2,1}, \ldots, P_{2, k-2}$, где

$$
P_{2, j}=\left\{c \in P_{2}: c \mid q^{2^{j} m_{1}}-1, c \nmid q^{r}-1, r<2^{j} m_{1}\right\}
$$

Из определения множеств $P_{2, j}$ видно, что все делители числа $q^{2^{j}} m_{1}-1$, не делящие $q^{r}-1$ при $r<2^{j} m_{1}$, исчерпываются числами

$$
\begin{aligned}
2^{e_{0}+j} c, & c \in P_{2,0} \cup P_{2,1} \cup \ldots \cup P_{2, j-1}, \\
2^{t} c, & c \in P_{2, j}, \quad t=0, \ldots, e_{0}+j,
\end{aligned}
$$


где $e_{0}=\exp _{2}(q-1)$. Обозначив множество всех этих чисел через $D_{j}$, получаем, что

$$
2^{j} m_{1} \Phi\left(2^{j} m_{1}\right)=\sum_{x \in D_{j}} \phi(x)
$$

или, подробнее,

$$
\begin{aligned}
2^{j} m_{1} \Phi\left(2^{j} m_{1}\right)=\sum_{c \in P_{2,0}} \phi\left(2^{e_{0}+j} c\right) & +\sum_{c \in P_{2,1}} \phi\left(2^{e_{0}+j} c\right)+\ldots+\sum_{c \in P_{2, j-1}} \phi\left(2^{e_{0}+j} c\right) \\
& +\sum_{c \in P_{2, j}} \phi(c)+\sum_{c \in P_{2, j}} \phi(2 c)+\ldots+\sum_{c \in P_{2, j}} \phi\left(2^{e_{0}+j} c\right) .
\end{aligned}
$$

Суммируя эти равенства по всем $j=0,1, \ldots, k-2$, получаем, что

$$
\sum_{j=0}^{k-2} 2^{j} m_{1} \Phi\left(2^{j} m_{1}\right)=2^{e_{0}+k-2} \sum_{c \in P_{2}} \phi(c)=2^{e_{0}+k-2} S_{2} .
$$

Отсюда находим, что

$$
S_{2}=\frac{1}{2^{e_{0}+k-2}} \sum_{j=0}^{k-2} 2^{j} m_{1} \Phi\left(2^{j} m_{1}\right)
$$

или, учитывая, что $m=2^{k} m_{1}$,

$$
S_{2}=\frac{1}{2^{e_{0}+k-2}} \sum_{i=2}^{k} \frac{m}{2^{i}} \Phi\left(\frac{m}{2^{i}}\right) .
$$

Теперь, в зависимости от значения величины $h$ в формуле (14), рассмотрим два случая.

Пусть в первом случае $m / 4$ четно или $\exp _{2}(q-1)>1$. Тогда согласно лемме 1

$$
e=\exp _{2}\left(2\left(q^{m / 2}-1\right)\right)=h+2,
$$

то есть $h=e-2$, и формула (14) принимает вид

$$
\frac{m}{2} \Phi\left(\frac{m}{2}\right)=2^{e-1} S_{1}+2^{e-2} S_{2}
$$

Следовательно,

$$
S_{1}+\frac{1}{2} S_{2}=\frac{m}{2^{e}} \phi\left(\frac{m}{2}\right)
$$

и значит,

$$
S_{1}+S_{2}=\left(S_{1}+\frac{1}{2} S_{2}\right)+\frac{1}{2} S_{2}=\frac{m}{2^{e}} \phi\left(\frac{m}{2}\right)+\frac{1}{2^{e_{0}+k-1}} \sum_{i=2}^{k} \frac{m}{2^{i}} \Phi\left(\frac{m}{2^{i}}\right)
$$

Из леммы 1 видно, что в рассматриваемом случае

$$
e=\exp _{2}\left(2\left(q^{m / 2}-1\right)\right)=\exp _{2}(q-1)+k=e_{0}+k .
$$


Поэтому

$$
S_{1}+S_{2}=\frac{1}{2^{e_{0}-1}} \sum_{i=1}^{k} \frac{1}{2^{i}} \Phi\left(\frac{m}{2^{i}}\right) .
$$

Отсюда и из формулы (13) получаем (6).

Во втором случае $m / 4$ нечетно и $\exp _{2}(q-1)=1$. В силу леммы 1

$$
h=\exp _{2}\left(q^{m / 4}-1\right)=\exp _{2}(q-1)=e_{0}=1, \quad k=2
$$

и из (14) находим, что

$$
\begin{aligned}
& \frac{m}{2} \Phi\left(\frac{m}{2}\right)=2^{e-1} S_{1}+2^{e-1}\left(1-\frac{1}{2^{e-2}}\right) \\
& \frac{m}{2^{e}} \Phi\left(\frac{m}{2}\right)=S_{1}+\left(1-\frac{1}{2^{e-2}}\right) S_{2} .
\end{aligned}
$$

Отсюда и из (15) следует, что

$$
\begin{aligned}
S_{1}+S_{2} & =S_{1}+\left(1-\frac{1}{2^{e-2}}\right) S_{2}+\frac{1}{2^{e-2}} S_{2} \\
& =\frac{m}{2^{e}} \Phi\left(\frac{m}{2}\right)+\frac{1}{2^{e-1}} \sum_{i=2}^{k} \frac{m}{2^{i}} \Phi\left(\frac{m}{2^{i}}\right) \\
& =\frac{m}{2^{e-1}} \sum_{i=1}^{k} \frac{1}{2^{i}} \Phi\left(\frac{m}{2^{i}}\right)
\end{aligned}
$$

Теперь из (13) снова получаем (6). Теорема доказана.

Рассмотрим теперь вопрос о неприводимых делителях многочленов $g_{u}(x)$ над полем $F_{q^{n}}$.

Теорема 2. Если п нечетно, то естъ $u= \pm 1$, то любой неприводимый над полем $F_{q}$ делитель многочлена $g_{u}(x)$ является неприводимъм над полем $F_{q^{n}}$.

Если п четно, то есть $u= \pm 2$, то любой неприводимый над полем $F_{q}$ делитель степени $m$ многочлена $g_{u}(x)$ разлагается над полем $F_{q^{n}}$ в произведение двух неприводимых многочленов степени $m / 2$.

Доказательство. Докажем первое утверждение. Пусть $h(x) \in F_{q}[x], \operatorname{deg} h(x)=m$, $h(x) \mid g_{u}(x)$, и $\alpha$ - корень $h(x)$ в его поле разложения. Тогда $m$ - наименьшее натуральное число, при котором $\alpha^{q^{m}-1}=1$. Так как $F_{q} \subset F_{q^{n}}$, многочлен $g_{u}(x)$ можно рассматривать как многочлен над $F_{q^{n}}$. Пусть $m^{\prime}-$ наименьшее натуральное число, при котором $\alpha^{\left(q^{n}\right)^{m^{\prime}}-1}=1$. Из определений чисел $m$ и $m^{\prime}$ получаем, что $m \mid n m^{\prime}$. С другой стороны, согласно теореме $1 m \mid n+u$, и, следовательно, $(m, n)=1$ при $u= \pm 1$, и $(m, n)=2$ при $u= \pm 2$. Отсюда видно, что $m=m^{\prime}$ при $u= \pm 1$, и $m^{\prime}=m / 2$ при $u= \pm 2$. Значит, над полем $F_{q^{n}}$ элемент $\alpha$ является корнем неприводимого многочлена степени $m$ при нечетном $n$ и степени $m / 2$ при четном $n$. Теорема доказана.

Из теорем 1, 2 получаем следующее утверждение. 
Следствие 1. Число неприводимых над полем $F_{q^{n}}$ делителей степени $m$ многочлена $g_{u}(x)$ вычисляется по формуле (6), если п нечетно, и равно

$$
2 \sum_{i=1}^{k} \frac{1}{2^{i}} \Phi\left(\frac{m}{2^{i}}\right)
$$

если п четно.

Следствие 2. Пусть

$$
g_{1}(x)=x^{q^{(n-u) / 2}-1}+1, \quad g_{2}(x)=x^{q^{(n+u) / 2}-1}+1
$$

- многочлены над полем $F_{q}, q$ нечетно, $n>1, u=1$ или 2 в зависимости от четности $n$.

Eсли $n \equiv 1(\bmod 2)$ или $n \equiv 2(\bmod 4)$, то многочлены $g_{1}(x), g_{2}(x)$ взаимно просты и не имеют хорней в поле $F_{q^{n}}$.

Ecлu $n \equiv 0(\bmod 4)$ mo

$$
\left(g_{1}(x), g_{2}(x)\right)=x^{q-1}+1
$$

многочленъ $g_{1}(x), g_{2}(x)$ не имеют корней в поле $F_{q}$ и имеют $q-1$ общих корней в nоле $F_{q^{n}}$.

Доказательство. Если $h(x)$ неприводим над $F_{q}$ и $h(x) \mid g_{i}(x), i=1,2$, то по теореме 1 выполнены следующие условия:

$$
m|n-u, \quad m| n+u, \quad \nmid(n-u) / 2, \quad m \nmid(n+u) / 2 .
$$

Нетрудно видеть, что эти условия могут выполняться лишь во втором случае при $m=2$. При этом корни $\alpha$ многочлена $h(x)$ должны удовлетворять условию

$$
\alpha^{2(q-1)}=1, \quad \alpha^{q-1} \neq 1 .
$$

Таких элементов ровно $q-1$, это корни многочлена $x^{q-1}+1$. Следовательно, $h(x)$ делит $x^{q-1}+1$ и остается заметить, что каждый такой многочлен $h(x)$ разлагается в произведение двух линейных множителей в силу теоремы 2.

Информация о числе решений уравнения (1) и о структуре этих решений содержится в следующей теореме.

Теорема 3. Пусть $f(x)$ - многочлен вида (3) над полем $F_{q^{n}}, n>1, a+b=s$, $s \mid q-1, M_{0}$ - множество корней многочлена $f(x)$ в поле $F_{q^{n}} u M_{1}=F_{q^{n}} \backslash M_{0}$.

Тогда для $\alpha \in F_{q^{n}}$ уравнение (1) имеет в $F_{q^{n}}$ ровно $s$ решений вида $(\alpha, \beta)$, если $\alpha \in M_{1}$, и единственное решение $(\alpha, 0)$, если $\alpha \in M_{0}$.

Если $N_{q^{n}}$ - число всех решений уравнения (1), то

$$
N_{q^{n}}= \begin{cases}q^{n} s, & \text { если q нечетно, } n \equiv 1(\bmod 2) \text { или } n \equiv 2(\bmod 4), \\ \left(q^{n}-q+1\right) s+q-1, & \text { если } q \text { любое, } 4 \mid n, \\ \left(q^{n}-q^{2}+1\right) s+q^{2}-1, & \text { если } q, n \text { четны, } 4 \nmid n .\end{cases}
$$


Доказательство. Докажем первое утверждение. Известно (см., например [5]), что число $N_{t}$ решений уравнения $y^{s}=t$ над полем $F_{q^{n}}$ выражается формулой

$$
N_{t}=\sum_{\text {ind } \chi=s} \chi\left(\text { norm }_{n} t\right)
$$

где norm $n t=t \cdot t^{\dot{q}} \ldots t^{q^{n-1}}-$ относительная норма элемента $t$, а суммирование ведется по всем характерам группы $F_{q}^{*}$, порядки которых делят $s$. В [1] доказано, что для многочлена $f(x)$ вида (2), а значит, и для многочлена вида (3), в случае нечетного $q$ элемент norm $_{n} f(\alpha)$ при любом $\alpha \in F_{q^{n}}$ является $s$-й степенью некоторого элемента этого поля. Следовательно,

$$
\chi\left(\operatorname{norm}_{n} f(\alpha)\right)= \begin{cases}1, & \text { если } f(\alpha) \neq 0, \\ 0, & \text { если } f(\alpha)=0, \chi \neq \chi_{0} .\end{cases}
$$

Так как существует ровно $s$ различных мультипликативных характеров показателя $s$, один из которых равен единичному характеру $\chi_{0}$, уравнение $y^{s}=f(\alpha)$ имеет $s$ решений при $f(\alpha) \neq 0$ и одно решение при $f(\alpha)=0$. Этим первое утверждение при нечетном $q$ доказано.

Для четного $q$, как и в [1], пользуясь тождеством $x^{q^{n}}=x$ в поле $F_{q^{n}}$, получим, что

$$
\operatorname{norm}_{n}(f(x))=\prod_{i=1}^{(n+1) / 2}\left(x^{q^{i-1}}+x^{q^{(n-1) / 2}+i-1}\right)^{s} \prod_{i=1}^{(n-1) / 2}\left(x^{q^{i-1}}+x^{q^{(n+1) / 2}+i-1}\right)^{s} .
$$

Значит, и в этом случае каждый элемент $f(\alpha)$ является $s$-й степенью элемента из $F_{q^{n}}$. Поэтому первое утверждение справедливо и при четном $q$.

Второе утверждение при нечетном $q$ следует непосредственно из первого утверждения и следствия теорем 1-2.

Пусть $q$ четно. Элемент $\alpha$ поля $F_{q^{n}}$ является корнем многочлена $x^{q^{(n \pm u) / 2-1}}-1$ в том и только том случае, когда

$$
\alpha^{\left(q^{n}-1, q^{(n \pm u) / 2}-1\right)}-1=1 .
$$

Так как

$$
\left(q^{n}-1, q^{(n \pm u) / 2}-1\right)=q^{(n,(n \pm u) / 2)}-1
$$

и $(n,(n \pm u) / 2)$ равен 1 или 2 соответственно в случаях, где $n \equiv 1(\bmod 2)$ или $4 \mid n$, и где $n \equiv 2(\bmod 4)$.

В первом случае получаем, что $f(x)$ имеет $q-1$ корней в $F_{q^{n}}$ и все они есть корни многочлена $x^{q-1}-1$; во втором случае $f(x)$ имеет $q^{2}-1$ корней в $F_{q^{n}}$, и все они есть корни многочлена $x^{q^{2}-1}-1$. Отсюда и следует второе утверждение теоремы при четном $q$. Теорема доказана.

Из доказательства теоремы 3 получаем полезное для дальнейшего следствие.

Следствие 3. Пусть q четно, тогда многочлень

$$
x^{q^{(n+u) / 2}-1}-1, \quad x^{q^{(n-u) / 2}-1}-1
$$

имегот наибольший общий делитель, равньй $x^{q-1}-1$, если $n \equiv 1(\bmod 2)$ или $4 \mid n$, и равный $x^{q^{2}-1}-1$, если $n \equiv 2(\bmod 4)$. 


\section{3. О точках и показателях полей алгебраических функций}

Приведем сначала некоторые общие сведения о полях алгебраических функций (см., например, [7]). Зафиксируем поле алгебраических функций $F=K(x, y)$, заданное над полем $K$ уравнением (1). Условимся максимальные идеалы дискретных колец нормирования поля $F$ называть точками поля $F$ и обозначать буквой $Q$ с индексами или без. Поле $K(x, y)$ является алгебраическим расширением степени $s$ поля рациональных функций $K(x)$. Поэтому все точки поля $K(x, y)$ являются расширениями соответствующих точек поля $K(x)$. Если точка $Q$ есть расширение точки $P$, то пишут $Q \mid P$. Все точки поля $K(x)$ известны - это идеалы $P_{p(x)}$, порождаемые неприводимыми над $K$ многочленами $p(x)$, и бесконечная точка $P_{\infty}$,

$$
\begin{aligned}
P_{p(x)} & =\{a(x) / b(x): p(x) \mid a(x), p(x) \nmid b(x)\} \\
P_{\infty} & =\{a(x) / b(x): \operatorname{deg} a(x)<\operatorname{deg} b(x)\} .
\end{aligned}
$$

Соответствующие им показатели для ненулевых элементов вычисляются по формулам

$$
\begin{aligned}
v_{P_{p(x)}}(c(x) / d(x)) & =\exp _{p(x)} c(x)-\exp _{p(x)} d(x) \\
v_{P_{\infty}}(c(x) / d(x)) & =\operatorname{deg} d(x)-\operatorname{deg} c(x)
\end{aligned}
$$

Кольцо дискретного нормирования $O_{w}$, где $w=p(x)$ или $\infty$, состоит из элементов $z \in K(x)$ с показателем $v_{P_{w}} \geqslant 0, \quad O_{w} / P_{w}=F_{w}$ - поле, содержащее $K$ (при отождествлении $\left.r \in K \operatorname{cr}+P_{w}\right),\left[F_{w}: K\right]=\operatorname{deg} P_{w}-$ степень точки $P_{w} ; \operatorname{deg} P_{p(x)}=\operatorname{deg} p(x)$ и $\operatorname{deg} P_{\infty}=1$. Таким образом, поле $K(x)$ содержит $q+1$ точек степени 1 - это точки $P_{x-\alpha}, \alpha \in K$, и $P_{\infty}$. Эти точки играют при построении кодов особую роль в силу того, что в этом случае поле $F_{w}$ изоморфно $K$ и смежный класс $z+P_{w}$ есть элемент из $K$; его называют значением элемента $z$ в точке $P_{w}$ и обозначают через $z\left(P_{w}\right)$. Следуя [7], далее точки $P_{x-\alpha}$ будем обозначать $P_{\alpha}$.

Если точка $Q$ поля $F$ является расширением точки $P$ поля $K(x)$, то для любого элемента $z \in K(x)$

$$
v_{Q}(z)=e(Q \mid P) v_{P}(z)
$$

где $e(Q \mid P)$ - индекс ветвления точки $P$ при переходе от $K(x)$ к $F$.

Дивизором поля $F$ называют формальную линейную комбинацию точек поля $F$ над Z с конечным числом неравных нулю коэффициентов. Если

$$
D=\sum_{P \in S} r_{P} P
$$

- дивизор и $r_{P} \geqslant 0$ для всех $P \in S$, то пишут $D \geqslant 0$, число $\sum_{P \in S} r_{P} \operatorname{deg} P$ называют степенью дивизора $D$ и обозначают $\operatorname{deg} D$. Каждому элементу $z \in F$ сопоставляют дивизор

$$
(z)=\sum v_{Q}(z) Q
$$

где сумма берется по всем точкам $Q$ с условием $v_{Q}(z) \neq 0$. Такие дивизоры называются главными. При построении кодов Гоппы необходимо иметь определенную 
информацию о точках поля $K(x, y)$, их степенях, индексах ветвления, соответствующих показателях элементов и т.д. Далее, как и раньше, будем считать, что $K=F_{q^{n}}$, а $f(x)$ имеет вид (3).

Из следствия 2 теорем 1,2 и следствия 3 теоремы 3 видно, что свойства кривой (1) с многочленом $f(x)$ вида (3) существенно различаются в следующих трех случаях:

1) $q$ нечетно, $n$ нечетно или $n \equiv 2(\bmod 4)$;

2) $q$ любое, $4 \mid n$;

3) $q$ четное, $n$ четное, $4 \nmid n$.

Кривая (1) в случае 1) несингулярна, а в случаях 2) и 3) сингулярна. Последнее имеет своей причиной наличие у многочлена $f(x)$ корней в поле $F_{q^{n}}$. В связи с этим в случаях 2) и 3) мы вместо многочлена (3) $f(x)=f_{1}(x)^{a} f_{2}(x)^{b}$ будем рассматривать соответственно многочлены

$$
g(x)=g_{1}(x)^{a} g_{2}(x)^{b}, \quad h(x)=h_{1}(x)^{a} h_{2}(x)^{b},
$$

где

$$
\begin{array}{ll}
g_{1}(x)=\frac{x^{q^{(n-u) / 2}-1}+1}{x^{q-1}-1}, & g_{2}(x)=\frac{x^{q^{(n+u) / 2}-1}+1}{x^{q-1}-1}, \\
h_{1}(x)=\frac{x^{q^{(n-u) / 2}-1}+1}{x^{q^{2}-1}-1}, & h_{2}(x)=\frac{x^{q^{(n+u) / 2}-1}+1}{x^{q^{2}-1}-1} .
\end{array}
$$

Введем для общности дальнейших рассмотрений обозначение $\phi_{i}(x)$, полагая $\phi_{i}(x)$ в случаях 1), 2), 3) равной соответственно $f_{i}(x), g_{i}(x), h_{i}(x), i=1,2$.

Заметим, что в любом из этих случаев многочлен $f(x)=\phi_{1}(x)^{a} \phi_{2}(x)^{b}$ не имеет корней в $F_{q^{n}}$, а уравнение (1) имеет ровно $s q^{n}$ решений $(\alpha, \beta)$.

Теорема 4. Пусть $K=F_{q^{n}}, K(x, y)$ - поле алгебраических функций, заданное уравнением (1), где

$$
f(x)=\phi_{1}(x)^{a} \phi_{2}(x)^{b}, \quad a+b=s, \quad s \mid q-1, \quad(a, s)=1 .
$$

Toгda

1) $K(x, y)$ есть циклическое расширение поля $K(x)$ степени $s, K$ алгебраически замкнуто в $K(x, y)$, и род поля $K(x, y)$ равен

$$
g=\frac{s-1}{2}\left(\operatorname{deg} \phi_{1}(x)+\operatorname{deg} \phi_{2}(x)-2\right),
$$

2) для каждой точки $P_{\alpha}$ поля $K(x)$ существует ровно $s$ расиирений $Q_{\alpha, \beta}$, где $\beta$ пробегает все решения уравнения $y^{s}=f(\alpha)$ в $K, Q_{\alpha, \beta}$ как идеал порождаетсл многочленом $x-\alpha$ и содержит $y-\beta, e\left(Q_{\alpha, \beta} \mid P_{\alpha}\right)=1, \operatorname{deg} Q_{\alpha, \beta}=\operatorname{deg} P_{\alpha}=1$,

3) точха $P_{\infty}$ поля $K(x)$ имеет единственное расширение $Q_{\infty}$, порождаемое ках идеал элементом $x^{-1}, e\left(Q_{\infty} \mid P_{\infty}\right)=1, \operatorname{deg} Q_{\infty}=s$, 
4) если $p(x)$ - неприводимъй над $K$ многочлен и $p(x) \mid f(x)$, то для точки $P_{p(x)}$ поля $K(x)$ существует единственное расиирение $Q_{p(x)}$,

$$
\operatorname{deg} Q_{p(x)}=\operatorname{deg} P_{p(x)}=\operatorname{deg} p(x), \quad e\left(Q_{p(x)} \mid P_{p(x)}\right)=s,
$$

5) если $p(x)$ неприводим над $K$ и $p(x) \nmid f(x)$, то существует единственное расширение $Q_{p(x)}$ точки $P_{p(x)} u$

$$
e\left(Q_{p(x)} \mid P_{p(x)}\right)=1, \quad \operatorname{deg} Q_{p(x)}=\operatorname{deg} P_{p(x)}=\operatorname{deg} p(x) .
$$

Доказательство. Докажем утверждение части 1). Так как $s \mid q-1$, то $(s, \operatorname{char} K)=1$, и $K$ содержит примитивный корень $s$-й степени из 1 . Кроме того, из условия $(a, s)=1$ следует, что $\left(s, \exp _{p(x)} f(x)\right)=1$ для любого неприводимого делителя $p(x)$ многочлена $f(x)$ над полем $K$. Следовательно, выполнены все условия утверждения 6.3.1 из [7]. Из него следует утверждение части 1) теоремы, а также равенства

$$
e(Q \mid P)= \begin{cases}s, & \text { если } P=P_{p(x)} \text { и } p(x) \mid f(x) \\ 1 & \text { в остальных случаях. }\end{cases}
$$

Докажем утверждение части 2). Согласно теореме $1 f(\alpha)$ при любом $\alpha \in K^{*}$ является $s$-й степенью подходящего элемента из $K$. Следовательно, с учетом условия $s \mid q-1$, уравнение $y^{s}=f(\alpha)$ имеет ровно $s$ различных решений. То же самое, очевидно, верно и при $\alpha=0$. Отсюда видно, что многочлен $z^{s}-f(\alpha)$ разлагается над $K$ в произведение $s$ различных линейных множителей. Кроме того, для любой точки $P$ поля $K(x)$, не равной $P_{\infty}$, справедливо неравенство $v_{P}(f(x)) \geqslant 0$ и, значит, $y$ является целым элементом кольца $O_{P}$. Таким образом, выполнены все условия теоремы Куммера 3.3.7 из [7]. Из нее и ее следствия 3.3.8 получаем утверждение части 2).

Для доказательства части 3) воспользуемся соответствиями между полями алгебраических функций и проективными кривыми. Полю $K(x, y)$ соответствует гладкая проективная кривая с уравнением

$$
z^{t}\left(y^{s}-f(1 / z)\right)=0
$$

где $t=\operatorname{deg} f(x)$. При $z=0$ получаем $x^{t}=0$ и, значит, на кривой (18) лежит лишь одна бесконечная точка $(0: 1: 0)$. Следовательно, для точки $P_{\infty}$ существует единственное расширение $Q_{\infty}$. Рассмотрим на $K(x, y)$ главный дивизор $(x)$. Из утверждения части 2) с учетом равенства (17) получаем, что

$$
(x)=P_{0, \beta_{1}}+\ldots+P_{0, \beta_{s}}-Q_{\infty} .
$$

Отсюда по теореме 1.4.11 из [7] и утверждению части 2) получаем, что

$$
\operatorname{deg} Q_{\infty}=\sum_{i=1}^{s} \operatorname{deg} P_{0, \beta_{i}}=s
$$

Порождение $Q_{\infty}$ как идеала в соответствующем кольце дискретного нормирования $O_{Q_{\infty}}$ элементом $x^{-1}$ следует из условия $v_{Q_{\infty}}(1 / x)=1$. 
В случае 4) $P=P_{p(x)}, p(x) \mid f(x)$. Из теоремы Куммера получаем, что существует $Q \mid P_{p(x)}$ такое, что $y \in Q$. Значит, $v_{Q}(y) \geqslant 1$. С другой стороны,

$$
v_{Q}(y)=\frac{1}{s} v_{Q}\left(y^{s}\right)=\frac{1}{s} e\left(Q \mid P_{p(x)}\right) v_{P_{p(x)}}\left(y^{s}\right)=\frac{1}{s} e\left(Q \mid P_{p(x)}\right) v_{P_{p(x)}}(f(x)) .
$$

Так как $p(x) \mid f(x)$ и $\exp p(x)=a$ или $b$, справедливы равенства

$$
v_{P_{p(x)}}= \begin{cases}a, & \text { если } p(x) \mid \phi_{1}(x), \\ b, & \text { если } p(x) \mid \phi_{2}(x) .\end{cases}
$$

Следовательно,

$$
v_{Q}(y)= \begin{cases}(a / s) e\left(Q \mid P_{p(x)}\right), & \text { если } p(x) \mid \phi_{1}(x) \\ (b / s) e\left(Q \mid P_{p(x)}\right), & \text { если } p(x) \mid \phi_{2}(x)\end{cases}
$$

Так как $v_{Q}(y) \geqslant 1, a / s<1, b / s<1$, справедливо неравенство

$$
e\left(Q \mid P_{p(x)}\right)>\max \{s / a, s / b\}
$$

Если бы существовала еще одна точка $Q^{\prime} \mid P_{p(x)}$, то из тех же соображений выполнялось бы неравенство

$$
e\left(Q^{\prime} \mid P_{p(x)}\right)>\max \{s / a, s / b\}
$$

А так как, согласно теореме 3.1.11 из [7]

$$
\sum_{Q^{\prime} \mid P_{p(x)}} e\left(Q^{\prime} \mid P_{p(x)}\right) f\left(Q^{\prime} \mid P_{p(x)}\right)=s,
$$

получаем, что $P_{p(x)}$ имеет единственное расширение $Q_{p(x)}$.

Пусть $e\left(Q_{p(x)} \mid P_{p(x)}\right)=e, f\left(Q_{p(x)} \mid P_{p(x)}\right)=f$. Тогда $e f=s$ и $\operatorname{con}\left(P_{p(x)}\right)=e Q_{p(x)}$. По следствию 3.1.13 из [7]

$$
\begin{aligned}
\operatorname{deg}\left(e Q_{p(x)}\right) & =s \operatorname{deg}\left(P_{p(x)}\right)=s \operatorname{deg}(p(x)) \\
e \operatorname{deg}\left(Q_{p(x)}\right) & =s \operatorname{deg}(p(x)) .
\end{aligned}
$$

Отсюда и из (17) получаем утверждение части 4).

В случае 5) $P=P_{p(x)}, p(x) \nmid f(x)$. Пусть $Q-$ расширение $P_{p(x)}$. Справедливо равенство

$$
v_{Q}(y)=\frac{1}{s} v_{Q}\left(y^{s}\right)=\frac{1}{s} e(Q \mid P) v_{P}(f(x))=0
$$

(так как $p(x) \nmid f(x)$ ). Следовательно, $y \in O_{Q}$ и $y \notin Q$. Пусть $Q_{1}, \ldots, Q_{r}$ - все расширения точки $P$. Найдем главный дивизор $(p(x))$. В силу того, что $e\left(Q_{i} \mid P\right)=1$ и $e\left(Q_{\infty} \mid P_{\infty}\right)=1$

$$
(p(x))=v_{P}(p(x)) Q_{1}+\ldots+v_{P}(p(x)) Q_{r}+v_{P_{\infty}}(p(x)) Q_{\infty}
$$

Следовательно,

$$
(p(x))=Q_{1}+\ldots+Q_{r}-\operatorname{deg} p(x) Q_{\infty}
$$


Tак как $\operatorname{deg} p(x)=0$ (теорема 1.4.11, [7]),

$$
\sum_{i=1}^{r} \operatorname{deg} Q_{i}=\operatorname{deg} p(x) .
$$

Однако поле $O_{P} / P=F_{P}$ вложимо в поле $O_{Q_{i}} / Q_{i}$ и значит, $\operatorname{deg} Q_{i} \geqslant \operatorname{deg} P_{i}$. Из последнего равенства следует, что $r=1$ и $\operatorname{deg} Q_{i}=\operatorname{deg} p(x)$. Таким образом, существует единственное расширение $Q_{p(x)}$, и

$$
e\left(Q_{p(x)} \mid P_{p(x)}=1, \quad \operatorname{deg} Q_{p(x)}=\operatorname{deg} P_{p(x)}=\operatorname{deg} p(x) .\right.
$$

Теорема доказана.

Следствие 4. Пусть $Q$ - точка поля $K(x, y)$. Тогда если $z=u(x) / v(x)$, где $u(x), v(x) \in K[x]$, mo

$$
\begin{aligned}
& v_{Q}(z)= \begin{cases}\exp _{p(x)} u(x)-\exp _{p(x)} v(x), & \text { ecлu } Q=Q_{p(x)}, p(x) \nmid f(x), \\
s\left(\exp _{p(x)} u(x)-\exp _{p(x)} v(x)\right), & \text { еслu } Q=Q_{p(x)}, p(x) \mid f(x), \\
\operatorname{deg}(v(x))-\operatorname{deg}(u(x)), & \text { еслu } Q=Q_{\infty},\end{cases} \\
& v_{Q}(y)= \begin{cases}0, & \text { еслu } Q=Q_{p(x)}, p(x) \nmid f(x), \\
a, & \text { еслu } Q=Q_{p(x)}, p(x) \mid \phi_{1}(x), \\
b, & \text { еслu } Q=Q_{p(x)}, p(x) \mid \phi_{2}(x), \\
-s^{-1} \operatorname{deg} f(x), & \text { еслu } Q=Q_{\infty} .\end{cases}
\end{aligned}
$$

\section{4. О кодах Гоппы}

Пусть $K=F_{q^{n}}, F=K(x, y)$ - поле алгебраических функций, заданное уравнением (1) с $f(x)=\phi_{1}(x)^{a} \phi_{2}(x)^{b}, D, G$ - дивизоры поля $F$, причем $D=Q_{1}+\ldots+Q_{N}-$ сумма точек первой степени, все точки с ненулевыми коэффициентами в $G$ отличны от $Q_{1}, \ldots, Q_{N}$, и

$$
L(G)=\{z \in F:(z)+G \geqslant 0\} \cup\{0\} .
$$

Под геометрическим кодом Гоппы, заданным дивизорами $D$ и $G$, понимают (см. [7]) код

$$
C(D, G)=\left\{\left(z\left(Q_{1}\right), \ldots, z\left(Q_{N}\right)\right): z \in L(G)\right\} .
$$

В общем случае известно (см. [7]), что он является линейным $[n, k, d]$-кодом над полем $K$ с параметрами

$$
k \geqslant \operatorname{deg} G+1-g, \quad d \geqslant N-\operatorname{deg} G .
$$

При этом, если выполняются неравенства

$$
2 g-2<\operatorname{deg} G<N
$$

то $k=\operatorname{deg} G+1-g$ (заметим, что здесь $k-$ размерность кода, $d-$ кодовое расстояние, $g$ - род поля $F$ ). Известно также, что при $\operatorname{deg} G<N$ отображение

$$
\psi: L(G) \rightarrow C(D, G)
$$


действующее по правилу $\psi(z)=\left(z\left(Q_{1}, \ldots, z\left(Q_{N}\right)\right)\right.$, является изоморфизмом линейньх пространств над $K$. Поэтому в этом случае задача нахождения порождающей матрицы кода сводится к нахождению базиса пространства $L(G)$. Всюду далее будем считать это условие выполненным.

Рассмотрим сначала самый простой случай, при котором $s=2, D=Q_{1}+\ldots+Q_{N}$ - сумма всех $2 q^{n}$ точек поля $F$ и $G=r Q_{\infty}$. Этот случай представляет особый интерес также и с точки зрения работы [6], где исследуется метод конструирования кодов в виде произведения кодов на гиперэллиптических кривых.

Теорема 5. Пусть $F=K(x, y)$ - поле алгебраических функций, заданное уравнением

$$
y^{2}=f(x), \quad f(x)=\phi_{1}(x) \phi_{2}(x),
$$

$2 \mid q-1, D=Q_{1}+\ldots+Q_{N}-$ сумма всех точех первой степени поля $F$ u $G=r Q_{\infty}$.

Тогда для любого $r=1,2, \ldots, N / 2-1 \operatorname{\kappa од~} C(D, G)$ являетсл $[n, k, d]$-кодом над $K$ с параметрами $k=2(r+1)-(1 / 2) \operatorname{deg} f(x), d \geqslant N-2 r$ nрu $r \leqslant(1 / 2) \operatorname{deg} f(x)-1$ $u d=N-2 r$ nрu $r>(1 / 2) \operatorname{deg} f(x)-1$.

Базисом этого кода является система векторов

$$
\left(z\left(Q_{1}\right), \ldots, z\left(Q_{N}\right)\right)
$$

где $z$ пробегает множество

$$
x^{0}, x^{1}, \ldots, x^{r}
$$

если $r \leqslant(1 / 2) \operatorname{deg} f(x)-1$, и множество

$$
x^{0}, x^{1}, \ldots, x^{r}, x^{0} y, x^{1} y, \ldots, x^{t-1} y,
$$

где $t=r+1-(1 / 2) \operatorname{deg} f(x)$, если $r>(1 / 2) \operatorname{deg} f(x)-1$.

Доказательство. Из условия $r<N / 2$ следует, что $\operatorname{dim} C(d, g)=\operatorname{dim} L(g)$. Поэтому будем искать базис пространства $L(G)$. Из определения $L(G)$ и из формул $(19),(20)$ легко следует, что $L(G)$ содержит элементы вида $x^{i} y^{j}$, если $0 \leqslant j<2$, $i+(j / 2) \operatorname{deg} f \leqslant r$, и не содержит элементы вида $u(x) y^{i} / w(x)$, где $(u(x), w(x))=1$ и $\operatorname{deg} w(x)>0$. Кроме того, так как $[F: K(x)]=2$, указанные элементы $x^{i} y^{j}$ линейно независимы над $K$. Отсюда следует, что система

$$
B=\left\{x^{i} y^{j}: 0 \leqslant j \leqslant 1, i+(j / 2) \operatorname{deg} f \leqslant r\right\}
$$

есть базис кода $C(D, G)$. В случае $r \leqslant(1 / 2) \operatorname{deg} f-1$ система $B$ совпадает с системой (23), в противном случае с системой (24). Следовательно, утверждение о размерности кода доказано и остается найти $d$. Рассмотрим элемент

$$
z=\prod_{i=1}^{r}\left(x-\alpha_{i}\right)
$$

где $\alpha_{1}, \ldots, \alpha_{r}-$ попарно различные элементы из $K$. Очевидно, что $z \in L(G)$ и $z(Q)=0$ для всех точек $Q$ вида $Q_{\alpha_{i}, \beta}$. Таких точек $2 r$, и значит, $d \leqslant N-2 r$. Пусть $r>(1 / 2) \operatorname{deg} f-1$. По теореме 12 род поля $g=(1 / 2) \operatorname{deg} f-1$, и значит, $r>g$. Тогда в силу (21)

$$
d \geqslant N-\operatorname{deg} G \geqslant N-2 r .
$$

Следовательно, $d=N-2 r$, и теорема доказана. 
Найдем порождающую матрицу кода $C(D, g)$. Упорядочим произвольно элементы поля $K$. Пусть

$$
K=\left\{\alpha_{1}, \ldots, \alpha_{M}\right\}, \quad M=N / 2=q^{n},
$$

и $\pm \beta_{i}$ - решения уравнения $y^{2}=f\left(\alpha_{i}\right), i=1, \ldots, q^{n}$. Теперь упорядочим точки первой степени следующим образом:

$$
Q_{\alpha_{1}, \beta_{1}}, Q_{\alpha_{2}, \beta_{2}}, \ldots, Q_{\alpha_{M}, \beta_{M}}, Q_{\alpha_{1},-\beta_{1}}, Q_{\alpha_{2},-\beta_{2}}, \ldots, Q_{\alpha_{M},-\beta_{M}}
$$

Из вида элементов системы (23) замечаем, что первые $r+1$ строк искомой матрицы $C$ образуют подматрицу $C^{\prime}=(A, A)$, где

$$
A=\left(\begin{array}{cccc}
1 & 1 & \ldots & 1 \\
\alpha_{1} & \alpha_{2} & \ldots & \alpha_{M} \\
\alpha_{1}^{2} & \alpha_{2}^{2} & \ldots & \alpha_{M}{ }^{2} \\
\ldots \ldots & \ldots \ldots & \ldots & \ldots . \\
\alpha_{1}{ }^{r} & \alpha_{2}{ }^{r} & \ldots & \alpha_{M}^{r}
\end{array}\right)
$$

В случае $r \leqslant(1 / 2) \operatorname{deg} f-1$ матрица $C^{\prime}$ и будет порождающей матрицей нашего кода.

Если $r>(1 / 2) \operatorname{deg} f-1$, то матрица $C$ будет иметь вид

$$
\left(\begin{array}{cc}
A & A \\
B & -B
\end{array}\right)
$$

где матрица $B$ имеет размеры $m \times M, m=r+1-1 / 2 \operatorname{deg} f(x)$, и ее строки получаются покомпонентным умножением первых $m$ строк матрицы $A$ на вектор $\left(\beta_{1}, \beta_{2}, \ldots, \beta_{M}\right)$.

Замечание 1. Соотношения (21) для параметров кода сохраняются и в случае, если в качестве точек $Q_{1}, \ldots, Q_{N}$ взять любые $N$ точек первой степени, лишь бы $N$ удовлетворяло неравенствам (22). В частности, если выбрать первые $M$ точек из (25), то получим код той же размерности с порождающей матрицей

$$
\left(\begin{array}{l}
A \\
B
\end{array}\right) \text {. }
$$

При $r \leqslant(1 / 2) \operatorname{deg} f-1$ это будут коды Рида-Соломона над полем $F_{q^{n}}$. Они являются так называемыми МДР-кодами (кодами с максимально достижимым расстоянием). При $r>(1 / 2) \operatorname{deg} f-1$, полученные коды не будут МДР-кодами, но по своим параметрам они будут к ним близки.

При построении кодов Гоппы чаще всего в качестве $Q_{1}, \ldots, Q_{N}$ берут все точки первой степени поля $F$, а в качестве $G$ дивизор $r Q_{\infty}$. Далее мы рассмотрим более общий случай.

Введем следующие обозначения. Пусть $p_{i 1}(x), \ldots, p_{i t_{i}}(x)$ - все неприводимые над $F_{q^{n}}$ делители многочлена $\phi_{i}(x), i=1,2, p_{31}(x), \ldots, p_{3 t_{3}}(x)$ - некоторый набор неприводимых над $F_{q^{n}}$ многочленов, не являющихся делителями многочлена $f(x)$, $c_{i}=\operatorname{deg} \phi_{i}(x), i=1,2, c=s^{-1} \operatorname{deg} f(x), m_{i k}=\operatorname{deg} p_{i k}(x)$. Будем предполагать, что $m_{3 k}>1, k=1, \ldots, t_{3}$. Из [7] известно, что $m_{1 k}>1$ при $k=1, \ldots, t_{1}, m_{2 k}>1$ при $k=1, \ldots, t_{2}$. Возьмем дивизоры

$$
G=\sum_{k=1}^{t_{1}} r_{1 k} Q_{1 k}+\sum_{k=1}^{t_{2}} r_{2 k} Q_{2 k}+\sum_{k=1}^{t_{3}} r_{3 k} Q_{3 k}+r Q_{\infty}
$$


где $r_{i k} \geqslant 0, t_{k} \geqslant 0, r>0, D=Q_{1}+\ldots+Q_{N}-$ сумма точек первой степени поля $F$, и найдем базис кода $C(D, G)$. Для этого построим базис $L(G)$.

Из утверждения 1) теоремы 4 следует, что любой элемент $z \in F$ однозначно представляется в виде

$$
u_{0}(x)+u_{1}(x) y+\ldots+u_{s-1}(x) y^{s-1}
$$

где $u_{i}(x) \in K(x)$. Выясним, когда $u_{i}(x) y^{i} \in L(G)$.

Лемма 3. Элемент поля $F$

$$
z=\frac{x^{i} y^{j}}{w(x)}, \quad i \geqslant 0, \quad 0 \leqslant j<s, \quad w(x) \in F_{q^{n}}[x],
$$

содержится в пространстве $L(G)$ в том и только том случае, когда выполняются следующие условия:

1) любой неприводимъй над $F_{q^{n}}$ делитель многочлена $w(x)$ совпадает $c$ одним из многочленов $p_{l, k}, l=1,2,3, k=1, \ldots, t_{l}$;

2) $\exp _{p_{1, k}(x)} w(x) \leqslant\left(a j+r_{1, k}\right) / s, k=1, \ldots, t_{1}$;

3) $\exp _{p_{2, k}(x)} w(x) \leqslant\left(b j+r_{2, k}\right) / s, k=1, \ldots, t_{2}$;

4) $\exp _{p_{3, k}(x)} w(x) \leqslant r_{3, k}, k=1, \ldots, t_{3}$;

5) $\operatorname{deg} w(x)-i-j c+r \geqslant 0$, гдe $c=-v_{Q_{\infty}}(y)=s^{-1} \operatorname{deg} f(x)$.

Доказательство. Согласно определению пространства $L(G)$ элемент $z \neq 0$ из $F$ содержится в $L(G)$ в том и только том случае, когда $(z)+G \geqslant 0$, то есть $v_{Q}(z)+v_{Q}(G) \geqslant 0$ для любой точки $Q$ поля $F$. Из формул (19), (20) замечаем, что условия леммы эквивалентны условию $v_{Q}(z)+v_{Q}(G) \geqslant 0$ соответственно для точек $Q_{p(x)}, p(x) \nmid f(x), p(x) \neq p_{k, l}(x), Q_{p_{1, k}}(x), Q_{p_{2, k}}(x), Q_{p_{3, k}}(x), Q_{\infty}$. Лемма доказана.

Заметим, что

$$
\operatorname{deg} G=\sum_{l, k} r_{l k} \operatorname{deg} Q_{l, k}+r \operatorname{deg} Q_{\infty}
$$

Отсюда, на основании теоремы 4 получаем, что

$$
M=\operatorname{deg} G=\sum_{k=1}^{t_{1}} r_{1 k} m_{1 k}+\sum_{k=1}^{t_{2}} r_{2 k} m_{2 k}+s \sum_{k=1}^{t_{3}} r_{3 k} m_{3 k}+s r .
$$

Пользуясь леммой 3 , выберем для каждого $j=0,1, \ldots, s-1$ унитарный многочлен $w(x)=w_{j}(x)$ наибольшей степени, удовлетворяющий первым четырем условиям этой леммы. Получим

$$
w_{j}(x)=\phi_{1}^{[a j / s]} \phi_{2}^{[b j / s]} g_{1 j} g_{2 j} g_{3 j}
$$

где

$$
\begin{gathered}
g_{1 j}=\prod_{k=1}^{t_{1}} p_{1 k}(x)^{\left[\left(a j+r_{1 k}\right) / s\right]-[a j / s]}, \quad g_{2 j}=\prod_{k=1}^{t_{2}} p_{2 k}(x)^{\left[\left(b j+r_{2 k}\right) / s\right]-[b j / s]}, \\
g_{3 j}=\prod_{k=1}^{t_{3}} p_{3 k}(x)^{r_{3 k}} .
\end{gathered}
$$


Последнее условие леммы 3 для числа $x^{i} y^{j} / w_{j}(x)$ запишется в виде $i \leqslant d_{j}$, где $d_{j}=\operatorname{deg} w_{j}(x)-j c+r$. Докажем, что при любом $j=0,1, \ldots, s-1$ выполняется неравенство $d_{j} \geqslant-1$. Из неравенства $2 g-2<\operatorname{deg} G$ (см. (22)) находим, что

$$
r \geqslant \frac{1}{s}\left((s-1)\left(c_{1}+c_{2}-2\right)-1-\sum_{k=1}^{t_{1}} r_{1 k} m_{1 k}-\sum_{k=1}^{t_{2}} r_{2 k} m_{2 k}\right)-\sum_{k=1} t_{3} r_{3 k} m_{3 k} .
$$

Следовательно,

$$
\begin{aligned}
d_{j}+1 \geqslant c_{1} & {\left[\frac{a j}{s}\right]+c_{2}\left[\frac{b j}{s}\right]+\sum_{k=1}^{t_{1}} m_{1 k}\left(\left[\frac{a j+r_{1 k}}{s}\right]-\left[\frac{a j}{s}\right]\right) } \\
& +\sum_{k=1}^{t_{2}} m_{2 k}\left(\left[\frac{b j+r_{2 k}}{s}\right]-\left[\frac{b j}{s}\right]\right)+\frac{(s-1)\left(c_{1}+c_{2}-2\right)-1}{s} \\
& -\frac{1}{s}\left(\sum_{k=1}^{t_{1}} r_{1 k} m_{1 k}+\sum_{k=1}^{t_{2}} r_{2 k} m_{2 k}\right)-j c+1 .
\end{aligned}
$$

Заметим, что

$$
c_{i}=\operatorname{deg} \phi_{i}(x)=\sum_{k=1}^{t_{i}} m_{i k}, \quad i=1,2, \quad c=\frac{1}{s}\left(a c_{1}+b c_{2}\right) .
$$

Поэтому неравенство (28) можно записать в виде

$$
d_{j+1} \geqslant \sum_{k=1}^{t_{1}} u_{1 k} m_{1 k}+\sum_{k=1}^{t_{2}} u_{2 k} m_{2 k}-\frac{s-1}{s} .
$$

При этом

$$
\begin{aligned}
& u_{1 k}=\left[\frac{a j+r_{1 k}}{s}\right]+\frac{s-1}{s}-\frac{r_{1 k}}{s}-\frac{j a}{s} \\
& u_{2 k}=\left[\frac{b j+r_{2 k}}{s}\right]+\frac{s-1}{s}-\frac{r_{2 k}}{s}-\frac{j b}{s} .
\end{aligned}
$$

Очевидно, что $u_{1 k} \geqslant 0, u_{2 k} \geqslant 0$ и, значит, $d_{j}+1 \geqslant-(s-1) / s$. Отсюда, учитывая, что число $d_{j}+1$ целое, получаем, что $d_{j}+1 \geqslant 0$, то есть $d_{j} \geqslant-1$.

Пусть

$$
B_{j}=\left\{\frac{x^{i} y^{j}}{w_{j}(x)}: 0 \leqslant i \leqslant d_{j}\right\},
$$

если $d_{j} \geqslant 0$, и $B_{j}=\varnothing$, если $d_{j}=-1$.

Теорема 6. Если для определенного равенством (26) дивизора $G$ виполняется условие (22), то множсество

$$
B=\bigcup_{j=0}^{s-1} B_{j}
$$

является базисом пространства $L(G)$. 
Доказательство. Из леммы 3 видно, что $B \subset L(G)$. Из однозначности представления элементов из $F$ в виде (27) следует, что система $B$ линейно независима над $F_{q^{n}}$. Остается доказать равенство $|B|=\operatorname{dim} L(G)$.

Так как $2 g-2<\operatorname{deg} G$, то, как следует из теоремы Римана-Роха (теорема I.5.17 B [7]),

$$
\begin{aligned}
\operatorname{dim} L(G) & =\operatorname{deg} G+1-g \\
& =\sum_{k=1}^{t_{1}} r_{1 k} m_{1 k}+\sum_{k=1}^{t_{2}} r_{2 k} m_{2 k}+s \sum_{k=1}^{t_{3}} r_{3 k} m_{3 k}+s r+1-\frac{s-1}{s}\left(c_{1}+c_{2}-2\right) .
\end{aligned}
$$

Найдем $|B|$. Из построения множества $B$ заключаем, что

$$
|B|=\sum_{j=0}^{s-1}\left(d_{j}+1\right)=\sum_{j=0}^{s-1}\left(\operatorname{deg} w_{j}(x)-j\left(a c_{1}+b c_{2}\right)+r+1\right) .
$$

Из (27) находим, что

$$
\begin{aligned}
\operatorname{deg} w_{j}(x)=c_{1}\left[\frac{a j}{s}\right]+c_{2}\left[\frac{b j}{s}\right]+\sum_{k=1}^{t_{1}} m_{1 k}\left(\left[\frac{a j+r_{1 k}}{s}\right]-\left[\frac{a j}{s}\right]\right) \\
+\sum_{k=1}^{t_{2}} m_{2 k}\left(\left[\frac{b j+r_{2 k}}{s}\right]-\left[\frac{b j}{s}\right]\right)+\sum_{k=1}^{t_{3}} r_{3 k} m_{3 k} .
\end{aligned}
$$

Воспользовавшись легко проверяемым равенством

$$
\sum_{j=0}^{s-1}\left[\frac{a j+r}{s}\right]=\frac{(a-1)(s-1)}{s}+r
$$

справедливым для любых целых чисел $a>0, s>0, r \geqslant 0, s \in \mathrm{N}$, при условии, что $(a, s)=1$, получаем, что

$$
\sum_{j=0}^{s-1} \operatorname{deg} w_{j}(x)=c_{1} \frac{(a-1)(s-1)}{2}+c_{2} \frac{(b-1)(s-1)}{2}+M
$$

где

$$
M=\sum_{k=1}^{t_{1}} r_{1 k} m_{1 k}+\sum_{k=1}^{t_{2}} r_{2 k} m_{2 k}+s \sum_{k=1}^{t_{3}} r_{3 k} m_{3 k}
$$

Следовательно,

$$
\begin{aligned}
B & =c_{1} \frac{(a-1)(s-1)}{2}+c_{2} \frac{(b-1)(s-1)}{2}+M-\frac{s-1}{s}\left(a c_{1}+b c_{2}\right)+r s+s \\
& =M-\frac{s-1}{s}\left(c_{1}+c_{2}\right)+s+r s=\operatorname{dim} L(G) .
\end{aligned}
$$

Теорема доказана. 
Для построения порождающей матрицы кода $C(D, G)$ упорядочим точки первой степени следующим образом:

$$
Q_{\alpha_{1}, \beta_{1}}, \ldots, Q_{\alpha_{q^{n}}, \beta_{q^{n}}}, Q_{\alpha_{1}, \beta_{1} \xi}, \ldots, Q_{\alpha_{q^{n}}, \beta_{q^{n}} \xi}, \ldots, Q_{\alpha_{1}, \beta_{1} \xi^{s-1}}, \ldots, Q_{\alpha_{q^{n}}, \beta_{q^{n}} \xi^{s-1}},
$$

где $\xi$ - любой элемент порядка $s$ в поле $F_{q^{n}}, \alpha_{1}, \ldots, \alpha_{q^{n}}$ - произвольная перестановка элементов поля $F_{q^{n}}$. Тогда при максимальном значении $N=q^{n} s$ порождающая матрица кода $C(D, G)$ будет иметь вид

$$
A=\left(\begin{array}{cccc}
A_{0} & A_{0} & \ldots & A_{0} \\
A_{1} & A_{1} \xi & \ldots & A_{1} \xi^{s-1} \\
A_{2} & A_{2} \xi & \ldots & A_{2} \xi^{s-1} \\
\ldots \ldots \ldots \ldots \ldots \ldots \ldots \ldots \ldots \ldots \ldots \\
A_{s-1} & A_{s-1} \xi & \ldots & A_{s-1} \xi^{s-1}
\end{array}\right)
$$

где $A_{j}$ - матрица размеров $\left(d_{j}+1\right) \times q^{n}$ и $A_{j}$ получается из первых $d_{j}+1$ строк матрицы $B$ их покомпонентным умножением на вектор

$$
\left(v_{j, 1}, \ldots, v_{j, q^{n}}\right),
$$

где $v_{j, k}=\beta_{k} w_{j}\left(\alpha_{k}\right)^{-1}, k=1, \ldots, q^{n}$, и

$$
B=\left(\begin{array}{cccc}
1 & 1 & \ldots & 1 \\
\alpha_{1} & \alpha_{2} & \ldots & \alpha_{q^{n}} \\
\alpha_{1}^{2} & \alpha_{2}^{2} & \ldots & \alpha_{q^{n}}^{2} \\
\ldots \ldots \ldots & \ldots \ldots \ldots \ldots & \ldots \ldots \\
\alpha_{1}^{q^{n}-2} & \alpha_{2}^{q^{n}-2} & \ldots & \alpha_{q^{n}}^{q^{n}-2}
\end{array}\right)
$$

Следствие 5. . Если дивизор $G$ имеет вид (26), $D$ - сумма всех точех первой степени поля $F$ и выполнено условие $(22)$, то $C(D, G)$ есть $[N, k, d]-$ код над полем $F_{q^{n}}$, гдe

$$
N=q^{n} s, \quad k=\operatorname{dim} C(D, G)=\operatorname{deg} G+1-g, \quad N-\operatorname{deg} G \leqslant d \leqslant N-s \max d_{j} .
$$

В этом утверждении доказательства требует лишь последнее неравенство. Для его доказательства достаточно заметить, что пространство, натянутое на $B_{j}$, содержит элементы вида $a(x) y^{j} / w_{j}(x)$, где $a(x)$ - любой многочлен степени $l \leqslant d_{j}$. Следовательно $L(G)$ содержит элемент

$$
\frac{1}{w_{j}(x)} \prod_{i=1}^{d_{j}}\left(x-\alpha_{i}\right) y^{j}
$$

который имеет значение 0 в $s d_{j}$ точках

$$
P_{\alpha_{i}, \beta_{i} \xi^{t}}, \quad i=1, \ldots, d_{j}, \quad t=0, \ldots, s-1,
$$

значит, $d \leqslant N-s d_{j}$.

Следствие 6. . Если $G=r Q_{\infty}, D-$ сумма всех точех первой степени поля $F u$

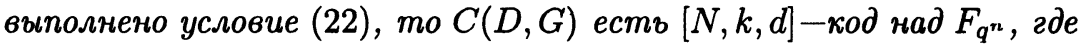

$$
N=q^{n} s, \quad k=\operatorname{deg} G+1-g, \quad d=N-\operatorname{deg} G .
$$


В этом случае последнее равенство следует из предыдущего следствия, если учесть, что $d_{0}=r$ и $d \leqslant N-s r=\operatorname{deg} G$ при $G=r Q_{\infty}$.

Замечание 2. Из этих двух следствий видно, что использование в качестве $G$ дивизоров, отличных от $r Q_{\infty}$, возможно позволит строить коды такой же размерности, что и при $G=r Q_{\infty}$, но с большим кодовым расстоянием.

\section{Список литературы}

1. Глухов М. М. (мл.), Нижние оценки сумм характеров от многочленов над конечными полями. Дискретная математика (1994) 6, №3, 136-142.

2. Глухов М. М. (мл.), О канонических разложениях некоторых двучленов над $\mathrm{GF}\left(q^{m}\right)$. Teзисы докл. II международной хонференции "Алгебрачческие, веролтностные, геометрические, комбинаторнъе и функциональные методы в теории чисел", Воронеж, 1995, c.41.

3. Лидл Р., Нидеррайтер Г., Конечные поля. Мир, Москва, 1988.

4. Ozbudak F., Glukhov M., Jr., Codes on superelliptic curves. Turkish J. Math (1998) 22, 223-234.

5. Степанов С. А., Арифметика алгебраических кривых. Наука, Москва, 1991.

6. Степанов С. А., Озбудак Ф., Расслоенные произведения гиперэллиптических кривых и геометрические коды Гоппы. Дискретная математика (1997) 9, №3, 36-42.

7. Stichtenoth H., Algebraic Function Fields and Codes. Springer, Berlin, 1993.

Статья поступила 26.06.2000. 